Tôhoku Math. Journ.

Vol. 19, No. 4, 1967

\title{
ON $C$-KILLING FORMS IN A COMPACT SASAKIAN SPACE
}

\author{
YôsuKe OGAwA
}

(Received July 10,1967)

Introduction. On a normal contact metric space (=Sasakian space), we studied in a former paper [4] $C$-harmonic forms which are in certain sense an extension of harmonic forms. There we defined two operators $\Gamma$ and $D$ which correspond to the exterior differential operator $d$ and the co-differential operator $\delta$ in a Riemann space. In this paper we get in the first place an integral formula for $\Gamma$ and $D$.

On the other hand it is well known that for any harmonic form in a compact Riemann space, the Lie derivative with respect to a Killing form always vanishes. In order to obtain its analogy for $C$-harmonic forms in a compact Sasakian space, we introduce the notion of $C$-Killing forms. Then we shall show as an application of the integral formula for $\Gamma$ and $D$ that for any $C$-harmonic form in a compact Sasakian space its Lie derivative with respect to a $C$-Killing form must be zero. Lastly we treat with the case of a compact regular Sasakian space.

We suppose that manifolds are connected and the differentiable structures are of class $C^{\infty}$.

I should like to express my hearty thanks to Professor S.Tachibana for his kind suggestions and many valuable criticisms.

1. Preliminaries. An $n$-dimensional Riemannian space $M^{n}$ is called a Sasakian space if it admits a unit Killing vector field $\eta^{\lambda}{ }^{1)}$ satisfying

$$
\nabla_{\lambda} \nabla_{\mu} \eta_{\nu}=\eta_{\mu} g_{\lambda v}-\eta g_{\lambda \mu}
$$

where $g_{\lambda_{\mu}}$ is the metric tensor of $M^{n}$. It is well known that $M^{n}$ is orientable and $n$ is odd. We put $\varphi_{\lambda_{\mu}}=\nabla_{\lambda} \eta_{\mu}, \varphi_{\lambda}{ }^{\mu}=\phi_{\lambda_{\rho}} g^{\rho \mu}$. Then there exist some wellknown identities, as follows (see Tachibana [2])

$$
\begin{gathered}
\nabla_{\lambda} \varphi_{\mu \nu}=\eta_{\mu} g_{\lambda_{\nu}}-\eta_{\nu} g_{\lambda_{\mu}}, \\
R_{\lambda \mu \nu \omega} \eta^{\omega}=\eta_{\lambda} g_{\mu \nu}-\eta_{\mu} g_{\lambda_{\nu}}, \\
\nabla^{\lambda} \varphi_{\lambda_{\mu}}=-(n-1) \eta_{\mu}, \quad \eta^{\lambda} R_{\lambda \mu}=(n-1) \eta_{\mu},
\end{gathered}
$$

1) The Greek indices $\lambda, \mu, \nu, \ldots$ run from 1 to $n$. 


$$
R_{\mu \rho} \boldsymbol{\varphi}_{\lambda}{ }^{\rho}=-R_{\lambda \rho} \varphi_{\mu}^{\rho}, \quad R_{\mu}^{\rho} \varphi_{\rho}^{\lambda}=R_{\rho}^{\lambda} \varphi_{\mu}{ }^{\rho} \text {. }
$$

We denote by $e(\eta)$ and $L$ (resp. $i(\eta)$ and $\Lambda$ ) the exterior product (resp. inner product) of 1-form $\eta$ and 2 -form $d \eta$, then we have [4]

$$
L=e(\eta) d+d e(\eta)
$$

$$
\Lambda=i(\eta) \delta+\delta i(\eta)
$$

We take an arbitrary 1 -form $\xi$. Then the inner product $\Lambda_{\xi}=i(d \xi)$ for any $p$-form $u=\left(u_{\lambda_{1} \ldots \lambda_{p}}\right)$ can be written by

$$
\left(\Lambda_{\xi} u\right)_{\lambda_{3} \ldots \lambda_{p}}=\nabla^{\rho} \xi^{\sigma} u_{\rho \sigma \lambda_{s} \ldots \lambda_{p}} \quad(p \geqq 2)
$$

and $\Lambda_{\xi} u=0$ if $p$ is 0 or 1 . We can easily obtain

$$
\Lambda_{\xi}=\delta i(\xi)+i(\xi) \delta
$$

The operators $\Phi, \nabla_{\eta}, \Gamma$ and $D$ for any $p$-form $u=\left(u_{\lambda_{1} \ldots \lambda_{p}}\right)$ are defined by

$$
\begin{aligned}
& (\Phi u)_{\lambda_{1} \cdots \lambda_{p}}=\sum_{i=1}^{p} \varphi_{\lambda_{i}}^{\sigma} u_{\lambda_{1} \cdots \hat{\sigma} \cdots \lambda_{p}}^{i}, \quad(p \geqq 1) \\
& \left(\nabla_{\eta} u\right)_{\lambda_{1} \ldots \lambda_{p}}=\eta^{\sigma} \nabla_{\sigma} u_{\lambda_{1} \ldots \lambda_{p}}, \quad(p \geqq 0) \\
& (\Gamma u)_{\lambda_{0} \ldots \lambda_{p}}=\sum_{\alpha=0}^{p}(-1)^{\alpha}{\phi_{\lambda}}_{\lambda_{\alpha}}^{\sigma} \nabla_{\sigma} u_{\lambda_{0} \ldots \hat{\alpha} \cdots \lambda_{p}}, \quad(p \geqq 0) \\
& (D u)_{\lambda_{2} \ldots \lambda_{p}}=\phi^{\sigma \rho} \nabla_{\sigma} u_{\rho \lambda_{2} \ldots \lambda_{p}}, \quad(p \geqq 1)
\end{aligned}
$$

where $u_{\lambda_{1} \ldots \hat{\sigma} \ldots \lambda_{p}}^{i}$ means the subscript $\sigma$ appears at the $i$-th position and $u_{\lambda_{0} \ldots \hat{\alpha} \ldots \lambda_{p}}$ means the $\alpha$-th subscript $\lambda_{\alpha}$ is omitted.

We denote by $\theta(\xi)$ the Lie derivative with respect to a vector field $\xi^{\lambda}$. For a 1 -form $\xi=\left(\xi_{\lambda}\right)$, identifying the covariant vector field with a contravariant vector field by the metric tensor, we also denote by $\theta(\xi)$ the Lie derivative of the vector field $\xi^{\lambda}=y^{\lambda \mu} \xi_{\mu}$.

Let the space $M^{n}$ be compact. Then the global inner product of any $p$-forms $u$ and $v$ is given by

$$
(u, v)=\int_{M^{n}} u \wedge * v
$$

where the notations $*$ and $\wedge$ represent the dual operator and exterior 
product respectively. The dual operator * satisfies

$$
\text { ** = identity. }
$$

A $p$ form $u$ on a Sasakian space is called to be $C$-harmonic if it satisfies

$$
d u=0, \quad \delta u=e(\eta) \Lambda u
$$

Then clearly $C$-harmonic 1 -forms are harmonic, and the converse is true. On $C$-harmonic forms, the following results are known [3], [4].

PROPOSITION 1.1. In a compact Sasakian space, any C-harmonic p-form $u(p \leqq(n-1) / 2)$ satisfies $i(\eta) u=0$.

PROPOSITION 1.2. In a compact Sasakian space, for any C-harmonic $p$-form $u(p \leqq(n-1) / 2) \Lambda u$ is also C-harmonic.

PROPOSITION 1.3. In a compact Sasakian space, a p.form $u(p \leqq(n-1) / 2)$ is $C$-harmonic if and only if it satisfies $i(\eta) u=0$ and $\triangle u=L \Lambda u$, where $\triangle$ is the Laplacian.

2. Integral formulas. In the following we consider a compact Sasakian space $M^{n}$. As for the operators $\Gamma$ and $D$, we know the following relations.

LEMMA 2.1. [4] In a Sasakian space, we have for any p-form $u$

$$
\begin{aligned}
& D u=\delta \nabla_{\eta} u-\nabla_{\eta} \delta u+(n-p) i(\eta) u, \\
& \Gamma u=d \nabla_{\eta} u-\nabla_{\eta} d u-p e(\eta) u .
\end{aligned}
$$

LemMA 2.2. In a Sasakian space, we have for any p-form $u$ and $q$ form $v$

$$
\begin{aligned}
& \Gamma(u \wedge v)=\Gamma u \wedge v+(-1)^{p} u \wedge \Gamma v, \\
& * \Gamma * v=(-1)^{p} D u .
\end{aligned}
$$

PROOF. Since it holds good for forms $u=\left(u_{\lambda_{1} \ldots \lambda_{p}}\right)$ and $v=\left(v_{\lambda_{1} \ldots \lambda_{q}}\right)$

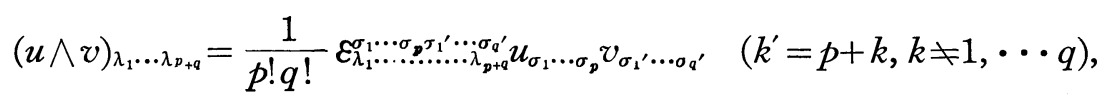

we have

$$
(\Gamma(u \wedge v))_{\lambda_{0} \ldots \lambda_{p+q}}=\phi_{\lambda_{0}^{\rho}}^{\rho} \nabla_{\rho}(u \wedge v)_{\lambda_{1} \ldots \lambda_{p+q}}-\sum_{i=1}^{p} \phi_{\lambda_{t}^{\rho}} \nabla_{\rho}(u \wedge v)_{\lambda_{1} \ldots \hat{\lambda}_{0} \ldots \lambda_{p} \lambda_{1} \cdots \lambda_{q^{\prime}}}^{i}
$$




$$
\begin{aligned}
& -\sum_{j=1}^{q} \varphi_{\lambda_{j^{\prime}}^{\rho}}^{\rho} \nabla_{\boldsymbol{p}}(u \wedge v)_{\lambda_{1} \ldots \lambda_{p} \lambda_{1^{\prime}} \ldots \hat{\lambda}_{0} \ldots \lambda_{\varphi^{\prime}}}^{\rho j}
\end{aligned}
$$

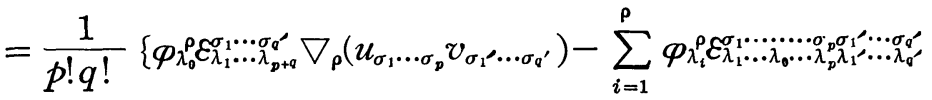

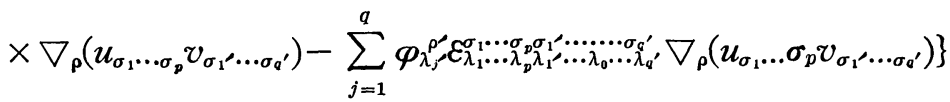

$$
\begin{aligned}
& =\frac{1}{p ! q !} \boldsymbol{\varphi}_{\sigma_{0}}^{\rho} \mathcal{E}_{\lambda_{0} \lambda_{1} \cdots \lambda_{p+q}}^{\sigma_{0} \sigma_{1} \cdots \sigma_{p+q}} \nabla_{\rho}\left(u_{\sigma_{1} \cdots \sigma_{p}} v_{\sigma_{1} \cdots \sigma_{q}}\right)
\end{aligned}
$$

On the other hand we have

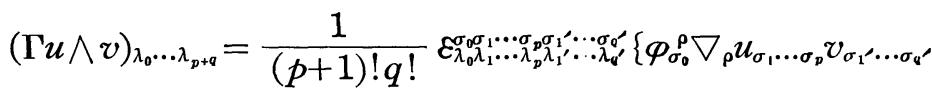

$$
\begin{aligned}
& \left.-\sum_{i=1}^{\rho} \varphi_{\sigma_{i}}^{\rho} \nabla_{\rho} u_{\sigma_{1} \cdots \hat{\sigma}_{0} \cdots \sigma_{q}}^{i} v_{\left.\sigma_{1} \cdots \sigma_{p}\right\}}\right\}
\end{aligned}
$$

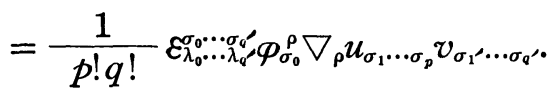

In the same way, we get

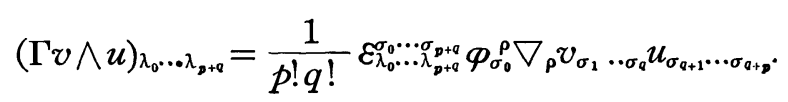

Therefore we have

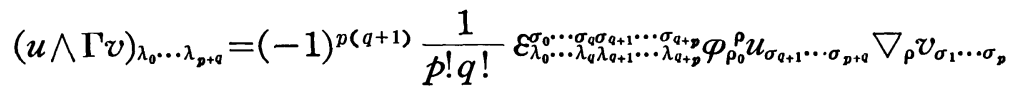

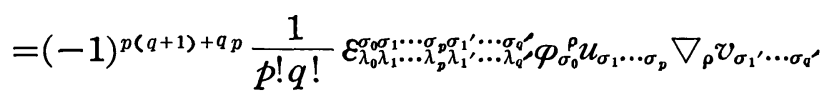

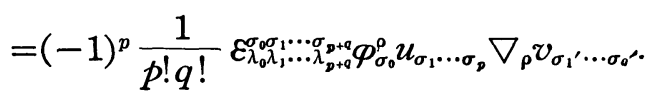

Thus we can obtain

$$
(\Gamma u \wedge v)_{\lambda_{0} \ldots \lambda_{p+q}}+(-1)^{p}(u \wedge \Gamma v)_{\lambda_{0} \ldots \lambda_{p+q}}=\frac{1}{p ! q !} \varepsilon_{\lambda_{0} \lambda_{1} \ldots \lambda_{p+q}}^{\sigma_{0} \sigma_{1} \cdots \sigma_{p+q}} \boldsymbol{\varphi}_{\sigma_{\bullet}}^{\rho}
$$




$$
\begin{aligned}
& \times\left(\nabla_{\rho} u_{\sigma_{1} \cdots \sigma_{q}} v_{\sigma_{1} \cdots \sigma_{q}}+u_{\sigma_{1} \cdots \sigma_{p}} \nabla_{\rho} v_{\sigma_{1} \cdots \sigma_{q}}\right) \\
& =\Gamma(u \wedge v)_{\lambda_{0} \cdots \lambda_{r+q^{*}}}
\end{aligned}
$$

Next we calculate (2.4). We write $g=\operatorname{det}\left(g_{\lambda_{\mu}}\right)$ and $\varepsilon_{\lambda_{1} \ldots \lambda_{n}}=\varepsilon_{\lambda_{1} \cdots \lambda_{n}}^{1 \cdots n}$. Then we have for any $p$ form $u$

$$
\begin{aligned}
& (* \Gamma * u)_{\lambda_{2} \cdots \lambda_{p}}=\frac{1}{(n-p+1) !} \sqrt{g} g^{\sigma_{0} \mu_{0}} \cdots g^{\sigma_{n-p} \mu_{n-p}}\left(\sum_{\alpha=0}^{n-p}(-1)^{\alpha} \phi_{\sigma_{\alpha}}^{\rho} \nabla_{\rho}(* u)_{\sigma_{0} \cdots \hat{\sigma}_{\alpha} \cdots \sigma_{n-p}}\right) \varepsilon_{\mu_{0} \cdots \mu_{n-p} \lambda_{2} \cdots \lambda_{p}} \\
& =\frac{1}{(n-p) !}(-1)^{\alpha+\alpha-1} \sqrt{g} g^{\sigma_{0} \mu_{0}} \cdots g^{\sigma_{n-p} \mu_{n-p}} \varphi_{\sigma_{0}}^{\rho} \nabla_{\rho}(* u)_{\sigma_{1} \cdots \sigma_{n-p}} \varepsilon_{\mu_{\alpha} \mu_{1} \cdots \hat{\mu}_{0} \cdots \mu_{n-p} \lambda_{2} \cdots \lambda_{p}} \\
& =\frac{g}{(n-p) ! p !} g^{\sigma_{1} \mu_{1}} \cdots g^{\sigma_{n-p} \mu_{n-p}} g^{\alpha_{1} \beta_{1}} \cdots g^{\alpha_{p} \beta_{p}} \mathcal{\rho}^{\mu_{\rho} \rho} \nabla_{\rho} u_{\beta_{1} \cdots \beta_{p} p} \varepsilon_{\alpha_{1} \cdots \alpha_{p} \sigma_{1} \cdots \sigma_{n-p}} \varepsilon_{\mu_{0} \cdots \mu_{n-p} \lambda_{2} \cdots \lambda_{p}} \\
& =\frac{1}{(n-p) ! p !}(-1)^{(n-p)(p-1)} \varepsilon^{\beta_{1} \cdots \beta_{p} \mu_{1} \cdots \mu_{n-p}} \varepsilon_{\mu_{0} \lambda_{2} \cdots \lambda_{p} \mu_{1} \cdots \mu_{n-p}} \phi^{\mu_{0} \rho} \nabla_{\rho} u_{\beta_{1} \cdots \beta_{p}} \\
& =\frac{1}{p !}(-1)^{n(p+1)} \varepsilon_{\mu_{0} \lambda_{2} \cdots \lambda_{p}}^{\beta_{1} \cdots \beta_{p}} \varphi^{\mu_{\rho} \rho} \nabla_{p} u_{\beta_{1} \cdots \beta_{p}} \\
& =(-1)^{n p+n+1}(D u)_{\lambda_{2}} \ldots \lambda_{p} \text {. }
\end{aligned}
$$

Since $n$ is odd, we have $(-1)^{n p+n+1}=(-1)^{p}$, hence $(2.4)$ is obtained.

LEMMA 2.3. In a Sasakian space, we have for any forms $u$ and $v$

$$
\begin{aligned}
& \nabla_{\eta}(u \wedge v)=\nabla_{\eta} u \wedge v+u \wedge \nabla_{\eta} v \\
& * \nabla_{\eta} * u=\nabla_{\eta} u
\end{aligned}
$$

PROOF. We take $u=\left(u_{\lambda_{1} \ldots \lambda_{p}}\right), v=\left(v_{\lambda_{1} \ldots \lambda_{q}}\right)$. Then

$$
\begin{aligned}
& \left(\nabla_{\eta}(u \wedge v)\right)_{\lambda_{1} \cdots \lambda_{p+q}}=\eta^{\rho} \nabla_{\rho}\left(\varepsilon_{\lambda_{1} \cdots \lambda_{p+q}^{1} \cdots \sigma_{p+q}}^{\sigma_{p+q}} u_{\sigma_{1} \cdots \sigma_{p}} v_{\sigma_{1}^{\prime} \cdots \sigma_{\rho}}\right) \\
& =\varepsilon_{\lambda_{1} \cdots \lambda_{p+q}}^{\sigma_{1} \cdots \sigma_{p+q}^{p+q}}\left(\eta^{\rho} \nabla_{\rho} u_{\sigma_{1} \cdots \sigma_{p}} v_{\sigma_{1^{\prime}} \cdots \sigma_{p^{\prime}}}+u_{\sigma_{1} \cdots \sigma_{p}} \eta^{\rho} \nabla_{\rho} v_{\sigma_{1^{\prime}} \cdots \sigma_{q^{\prime}}}\right) \\
& =\left(\nabla_{\eta} u \wedge v\right)_{\lambda_{1} \cdots p_{p+q}}+\left(u \wedge \nabla_{\eta} v\right)_{\lambda_{1} \cdots \lambda_{p+q}}
\end{aligned}
$$

and

$$
\left(* \nabla_{\eta} * u\right)_{\lambda_{1} \cdots \lambda_{p}}=\frac{1}{(n-p) !} \sqrt{g} g^{\sigma_{1} \mu_{1}} \cdots g^{\sigma_{n-p} \mu_{n-p} \eta^{\rho}} \nabla_{\rho}(* u)_{\sigma_{1} \cdots \sigma_{n-p}} \varepsilon_{\mu_{1} \cdots \mu_{n-p} \lambda_{1} \cdots \lambda_{p}}
$$




$$
\begin{aligned}
& =\frac{g}{(n-p) ! p !} g^{\sigma_{1} \mu_{1}} \cdots g^{\sigma_{n-p} \mu_{n-p}} g^{\alpha_{1} \beta_{1}} \cdots g^{\alpha_{p} \beta_{p}} \eta^{\rho} \nabla_{\rho} u_{\beta_{1} \cdots \beta_{p}} \varepsilon_{\alpha_{1} \cdots \alpha_{p} \tau_{1} \cdots \sigma_{n-p}} \varepsilon_{\mu_{1} \cdots \mu_{n-p} \lambda_{1} \cdots \lambda_{p}} \\
& =\frac{1}{(n-p) ! p !} \varepsilon^{\beta_{1} \cdots \beta_{p} \mu_{1} \cdots \mu_{n-p}} \varepsilon_{\mu_{1} \cdots \mu_{n-p} \lambda_{1} \cdots \lambda_{p}} \eta^{\rho} \nabla_{\rho} u_{\beta_{1} \cdots \beta_{p}} \\
& =(-1)^{p(n-p)}\left(\nabla_{\eta} u\right)_{\lambda_{1} \cdots \lambda_{p}} .
\end{aligned}
$$

As $n$ is odd, $(-1)^{p(n-p)}=1$ for any $p$, thus we get (2.6).

Since $\eta$ is a Killing form, the Lie derivative $\theta(\eta)$ with respect to $\eta$ satisfies the following relations [4].

$$
\begin{aligned}
& \theta(\boldsymbol{\eta})=-\delta e(\eta)-e(\boldsymbol{\eta}) \delta, \\
& * \theta(\boldsymbol{\eta}) * \theta(\boldsymbol{\eta}) .
\end{aligned}
$$

The operator $\Phi$ satisfies for any $u$

$$
\Phi u=\theta(\eta) u-\nabla_{\eta} u
$$

Therefore by virtue of Lemma 2.3 and the fact that $\theta(\boldsymbol{\eta})$ is a derivation, we have

LEMMA 2.4. [6] In a Sasakian space, we have for any forms $u$ and $v$

$$
\begin{aligned}
& \Phi(u \wedge v)=\Phi u \wedge v+u \wedge \Phi v, \\
& * \Phi * u=\Phi u .
\end{aligned}
$$

Using these Lemmas, we study some integral formulas in compact case.

THEOREM 2.5. In a compact Sasakian space, we have for any p-forms $u$ and $v$

$$
\begin{aligned}
& \left(\nabla_{\eta} u, v\right)=-\left(u, \nabla_{\eta} v\right), \\
& (\theta(\eta) u, v)=-(u, \theta(\eta) v), \\
& (\Phi u, v)=-(u, \Phi v) .
\end{aligned}
$$

PROOF. From (2.5) and (2.6) we have

$$
\nabla_{\eta} u \wedge * v=\nabla_{\eta}(u \wedge * v)-u \wedge * \nabla_{\eta} v
$$

As $u \wedge * v$ is an $n$ - form, there exists a function $f$ on $M^{n}$ such that $u \wedge * v$ is 
equal to $f \omega$, where $\omega$ is the volume element of $M^{n}$. It is known that $\omega$ is written as $c \eta \wedge(d \eta)^{m}(m=(n-1) / 2), c$ is a constant [5]. Since the relations

$$
\nabla_{\eta} \eta=0, \quad \nabla_{\eta} d \eta=0
$$

hold good, we have

$$
\nabla_{\eta}(u \wedge * v)=\nabla_{\eta} f \omega=-\delta(f \eta) \omega
$$

Hence we get

$$
\int_{M^{n}} \nabla_{\eta}(u \wedge * v)=0
$$

which means (2.10) is true. (2.11) is the result of (2.7) and

$$
\theta(\eta)=d i(\eta)+i(\eta) d
$$

(2.12) follows from (2.10), (2.11) and (2.8).

THEOREM 2.6. In a compact n-dimensional Sasakian space, we have for any $p$-form $u$ and ( $p+1)$-form $v$

$$
(\Gamma u, v)=(u, D v)-(n-1)(e(\eta) u, v) .
$$

PROOF. By virtue of Lemma 2.1 and Theorem 2.5, we see

$$
\begin{aligned}
(\Gamma u, v) & =\left(\nabla_{\eta} u, \delta v\right)-\left(\nabla_{\eta} d u, v\right)-p(u, i(\eta) v) \\
& =\left(u,-\nabla_{\eta} \delta v+\delta \nabla_{\eta} v-p i(\eta) v\right) \\
& =(u, D v-(n-p-1) i(\eta) v-p i(\eta) v) \\
& =(u, D v)-(n-1)(u, i(\eta) v) .
\end{aligned}
$$

This is the required result.

3. $\boldsymbol{C}$-Killing forms. Let $M^{n}$ be an $n$-dimensional Sasakian space. We call a 1 -form $\xi$ of $M^{n}$ to be $C$-Killing if it satisfies

$$
\begin{aligned}
& \delta \xi=0, \\
& \theta(\xi)\left(g_{\lambda \mu}-\eta_{\lambda} \eta_{\mu}\right)=0 .
\end{aligned}
$$

Clearly the 1 -form $\eta$ is $C$-Killing. The vector space of all vector fields identified 
with $C$-Killing forms is a Lie algebra. Especially we call a $C$-Killing form $\xi$ such that

$$
\xi^{\prime} \equiv i(\eta) \xi=\text { const. }
$$

to be special $C$-Killing. $\eta$ is a special $C$-Killing form. A Killing form which is at the same time a special $C$-Killing form is of the type $\xi^{\prime} \eta, \xi^{\prime}=$ const.

LEMMA 3.1. In a Sasakian space, we have for a C-Killing form

$$
\begin{aligned}
& \nabla_{\eta} \xi^{\prime}=0, \\
& \theta(\eta) \xi_{\lambda}=0 .
\end{aligned}
$$

PROOF. The equation (3.2) can be expressed as

$$
\nabla_{\lambda} \xi_{\mu}+\nabla_{\mu} \xi_{\lambda}=2 \xi^{\rho}\left(\varphi_{\rho \lambda} \eta_{\mu}+\varphi_{\rho \mu} \eta_{\lambda}\right)+\nabla_{\lambda} \xi^{\prime} \eta_{\mu}+\nabla_{\mu} \xi^{\prime} \eta_{\lambda}
$$

Transvecting (3.6) with $g^{\lambda_{\mu}}$, we obtain

$$
\delta \xi=-\nabla_{\eta} \xi^{\prime}
$$

hence (3.4) follows by virtue of (3.1). Next transvecting (3.6) with $\eta^{\lambda}$, we have

$$
\eta^{\lambda} \nabla_{\lambda} \xi_{\rho}+\varphi_{\rho}^{\lambda} \xi_{\lambda}=\left(\nabla_{\eta} \xi^{\prime}\right) \eta_{\rho}=0
$$

which means $\theta(\eta) \xi_{\lambda}=0$.

Let $\xi$ be a special $C$-Killing form. Then by virtue of (3.3) and (3.6) it satisfies

$$
\nabla_{\lambda} \xi_{\mu}+\nabla_{\mu} \xi_{\lambda}=2 \xi^{\rho}\left(\varphi_{\rho \lambda} \eta_{\mu}+\varphi_{\rho \mu} \eta_{\lambda}\right)
$$

Conversely, we show that (3.7) is a sufficient condition for a 1 -form $\xi$ to be special $C$-Killing, in a compact case. Evidently (3.1) follows from (3.7). Differentiating (3.7) by $\nabla^{\lambda}$, we have

$$
\nabla^{\lambda} \nabla_{\lambda} \xi_{\mu}+R_{\mu}^{\rho} \xi_{\rho}=-2 D \xi \eta_{\mu}+2 n \xi^{\prime} \eta_{\mu}-2 \xi_{\mu}+2 \eta^{\lambda} \nabla_{\lambda} \xi^{\rho} \boldsymbol{\varphi}_{\rho \mu}
$$

LEMMA 3.2. In a compact Sasakian space, for a 1-form $\xi$ satisfying (3.7) the scalar $\xi^{\prime}=i(\eta) \xi$ is a constant function. 
PROOF. Calculating the Laplacian of $\xi^{\prime}$, we have by virtue of (3.8)

$$
\begin{aligned}
\nabla^{\lambda} \nabla_{\lambda} \xi^{\prime} & =\eta^{u} \nabla^{\lambda} \nabla_{\lambda} \xi_{\mu}+2 D \xi-(n-1) \xi^{\prime} \\
& =-(n-1) \xi^{\prime}-2 D \xi+2 n \xi^{\prime}-2 \xi^{\prime}+2 D \xi-(n-1) \xi^{\prime} \\
& =0 .
\end{aligned}
$$

Therefore if $M^{n}$ is compact, $\xi^{\prime}$ must be constant.

From this Lemma 3.2, we see that the form $\xi$ having the property (3.7) also satisfies (3.6), and therefore it is a $C$-Killing form. Again from Lemma 3.2 it must be special $C$-Killing. Thus we proved the following

THEOREM 3.3. A 1-form $\xi$ on a compact Sasakian space is sfecial $C$-Killing if and only if it satisfies the relation (3.7).

Next we consider the relation between $C$-Killing and special $C$-Killing forms. Then

THEOREM 3.4. For a C-Killing form $\xi$ on a Sasakian space, a 1-form $\zeta$ defined by $\zeta=\xi-\xi^{\prime} \eta\left(\xi^{\prime}=i(\eta) \xi\right)$ is special C-Killing. Conversely for a special $C$-Killing form $\zeta$ and a scalar function $f, a_{0} 1$-form $\xi$ defined by $\xi=\xi+f \eta$ is $C$-Killing if and only if $\nabla_{\eta} f=0$.

PROOF. The first half. Since $\zeta^{\prime}=i(\eta) \zeta=0,(3.6)$ coincides with (3.7) for $\zeta$, hence we have only to show that $\zeta$ satisfies (3.7). Calculating directly, we get

$$
\begin{aligned}
\nabla_{\lambda} \zeta_{\mu}+\nabla_{\mu} \xi_{\lambda} & =\nabla_{\lambda} \xi_{\mu}+\nabla_{\mu} \xi_{\lambda}-\nabla_{\lambda} \xi^{\prime} \eta_{\mu}-\nabla_{\mu} \xi^{\prime} \eta_{\lambda}-\xi^{\prime} \boldsymbol{\varphi}_{\lambda \mu}-\xi^{\prime} \boldsymbol{\varphi}_{\mu \lambda} \\
& =2 \xi^{\rho}\left(\boldsymbol{\varphi}_{\boldsymbol{\rho} \lambda} \boldsymbol{\eta}_{\mu}+\boldsymbol{\varphi}_{\rho \mu} \boldsymbol{\eta}_{\lambda}\right) \\
& =2 \zeta^{\rho}\left(\boldsymbol{\varphi}_{\boldsymbol{\rho} \lambda} \boldsymbol{\eta}_{\mu}+\boldsymbol{\varphi}_{\boldsymbol{\rho} \mu} \boldsymbol{\eta}_{\lambda}\right) .
\end{aligned}
$$

For the latter half, we look for the condition that the 1 -form $f_{\eta}$ to be $C$ Killing, and get

$$
\begin{aligned}
& \delta(f \eta)=-\nabla_{\eta} f, \\
& \theta(f \eta)\left(g_{\lambda \mu}-\eta_{\lambda} \eta_{\mu}\right)=0 .
\end{aligned}
$$

Therefore if the 1 -form $\xi_{\lambda}$ is $C$-Killing, then $\nabla_{\eta} f=0$, and the converse is true.

4. Special $C$-Killing forms. In this section, we show that a $C$-harmonic $p$-form $(p \leqq(n-1) / 2)$ is invariant by a $C$-Killing form in a compact Sasakian 
space. Let $\xi$ be a special $C$-Killing form. Then we have from (3.8) and (3.5)

$$
\nabla^{\lambda} \nabla_{\lambda} \xi_{\mu}+R_{\mu \rho} \xi^{\rho}=-2(D \xi-(n+1) \xi) \eta_{\mu}-4 \xi_{\mu},
$$

where we put $\xi^{\prime}=i(\eta) \xi$. Differentiating (3.7) by $\nabla_{\nu}$ and adding cyclicly with respect to the subscript $\lambda, \mu, \nu$, we have

$$
\begin{aligned}
\nabla_{\lambda} \nabla_{\mu} \xi_{\nu} & =R_{\mu \nu \lambda}^{\varepsilon} \xi_{\varepsilon}+\eta_{\lambda}\left(\nabla_{\mu} \xi^{\rho} \boldsymbol{\varphi}_{\rho \nu}-\nabla_{\nu} \xi^{\rho} \boldsymbol{\varphi}_{\rho \mu}\right)+\eta_{\mu}\left(\nabla_{\lambda} \xi^{\rho} \boldsymbol{\varphi}_{\rho \nu}-\nabla_{\nu} \xi^{\rho} \boldsymbol{\varphi}_{\rho \lambda}\right) \\
& +\eta_{\nu}\left(\nabla_{\lambda} \xi^{\rho} \boldsymbol{\varphi}_{\rho \mu}+\nabla_{\mu} \xi^{\rho} \boldsymbol{\varphi}_{\rho \lambda}\right)+2 \xi^{\rho}\left(\boldsymbol{\varphi}_{\rho \lambda} \boldsymbol{\varphi}_{\mu \nu}+\boldsymbol{\varphi}_{\rho \mu} \boldsymbol{\varphi}_{\lambda \nu}\right) \\
& +2 \xi^{\prime} g_{\lambda \mu} \boldsymbol{\eta}_{\nu}+2\left(\boldsymbol{\eta}_{\lambda} \boldsymbol{\eta}_{\mu} \xi_{\nu}-\boldsymbol{\eta}_{\mu} \boldsymbol{\eta}_{\nu} \xi_{\lambda}-\eta_{\lambda} \eta_{\nu} \xi_{\mu}\right)
\end{aligned}
$$

which will be used in the proof of Lemma 4.2 .

By virtue of (2.8) and (3.5) we obtain

$$
\Phi \xi=-\nabla_{\eta} \xi(\equiv \bar{\xi}) .
$$

Then making use of (3.7), we have

$$
\begin{aligned}
(d \bar{\xi})_{\lambda_{\mu}} & =\nabla_{\lambda}\left(\varphi_{\mu \rho} \xi^{\rho}\right)-\nabla_{\mu}\left(\varphi_{\lambda_{\rho}} \xi^{\rho}\right) \\
& =\left(\eta_{\lambda} \xi_{\mu}-\eta_{\mu} \xi_{\lambda}\right)+\left(\varphi_{\lambda}^{\rho} \nabla_{\rho} \xi_{\mu}-\varphi_{\mu}^{\rho} \nabla_{\rho} \xi_{\lambda}\right) \\
& =(\boldsymbol{e}(\eta) \xi)_{\lambda_{\mu}}+(\Gamma \xi)_{\lambda_{\mu}}
\end{aligned}
$$

Thus we can get

$$
\overline{d \xi}=e(\eta) \xi+\Gamma \xi
$$

LEMMA 4.1. In a compact Sasakian space, for any 1-form satisfying $\xi^{\prime} \equiv i(\eta) \xi=$ constant we have

$$
(\Gamma \xi, e(\eta) \xi)=-(e(\eta) \xi, e(\eta) \xi)
$$

PROOF. In a Sasakian space, for any $p$-form $u$ the following

$$
\Gamma i(\eta) u+i(\eta) \Gamma u=-p u+e(\eta) i(\eta) u
$$

holds good. In fact, owing to (2.2) we have

$$
\begin{aligned}
\Gamma i(\eta) u+i(\eta) \Gamma u & =d i(\eta) \nabla_{\eta} u-\nabla_{\eta} d i(\eta) u-(p-1) e(\eta) i(\eta) u \\
& +i(\eta) d \nabla_{\eta} u-\nabla_{\eta} i(\eta) d u-p i(\eta) e(\eta) u
\end{aligned}
$$


$C$-KILLING FORMS IN A COMPACT SASAKIAN SPACE

$$
\begin{aligned}
& =\theta(\eta) \nabla_{\eta} u-\nabla_{\eta} \theta(\eta) u-p u+e(\eta) i(\eta) u \\
& =-p u+e(\eta) i(\eta) u,
\end{aligned}
$$

since $\nabla_{\eta}$ commutes with $i(\eta)$ and $\theta(\eta)$. Hence it follows for a 1 -form $\xi$

$$
\begin{aligned}
(e(\eta) \xi, \Gamma \eta) & =(\xi, i(\eta) \Gamma \xi) \\
& =(\xi,-\Gamma i(\eta) \xi-\xi+e(\eta) i(\eta) \xi) \\
& =(\xi,-\Gamma i(\eta) \xi-i(\eta) e(\eta) \xi) \\
& =\left(\xi, \Gamma \xi^{\prime}\right)-\left(e^{\prime}(\eta) \xi, e(\eta) \xi\right) .
\end{aligned}
$$

If $\xi^{\prime}$ is constant, then we have $\Gamma \xi^{\prime}=0$. This proves the lemma.

LEMMA 4.2. In a compact Sasakian space, we have for a special CKilling form $\xi$

$$
(\Gamma \xi, \Gamma \xi)=(e(\eta) \xi, e(\eta) \xi)
$$

PRoOF. Calculating $D \Gamma \xi$ for a special $C$-Killing form $\xi$, we first see from (4.2)

$$
\begin{aligned}
& \eta^{o} \eta^{\sigma} \nabla_{\rho} \nabla_{\sigma} \xi_{\lambda}=-\xi_{\lambda}+\xi^{\prime} \eta_{\lambda}, \\
& \varphi^{\rho \sigma} \nabla_{\rho} \nabla_{\tau} \xi_{\sigma}=\left(\varphi_{\rho}^{\sigma} R_{\sigma \tau}+(n+2) \boldsymbol{\varphi}_{\rho \tau}\right) \xi^{\rho} .
\end{aligned}
$$

Then making use of these relations and (4.1) we have

$$
\begin{aligned}
(D \Gamma \xi)_{\lambda} & =\phi^{\rho \sigma} \nabla_{\rho}\left(\varphi_{\sigma}{ }^{\top} \nabla_{\tau} \xi_{\lambda}-\varphi_{\lambda}{ }^{\top} \nabla_{\tau} \xi_{\sigma}\right) \\
& =-\nabla^{\rho} \nabla_{\rho} \xi^{\lambda}+\eta^{\top} \eta^{\sigma} \nabla_{\rho} \nabla_{\sigma} \xi_{\lambda}-D \xi \eta_{\lambda}+\varphi_{\lambda}{ }^{\rho} \eta^{\sigma} \nabla_{\rho} \xi_{\sigma}-\varphi_{\lambda}^{\tau} \phi^{\top} \nabla_{\rho} \nabla_{\tau} \xi_{\sigma} \\
& =-\nabla^{\rho} \nabla_{\rho} \xi_{\lambda}-R_{\lambda \rho} \xi^{\rho}-D \xi_{\lambda}-(n+2) \xi_{\lambda}+(2 n+1) \xi^{\prime} \eta_{\lambda} \\
& =D \xi \eta_{\lambda}-\xi^{\prime} \eta_{\lambda}-(n-2) \xi_{\lambda} .
\end{aligned}
$$

On the other hand, since $\Gamma \xi^{\prime}=0$ for a special $C$-Killing form $\xi_{\lambda}$, we have by virtue of (2.13)

$$
\left(D \xi, \xi^{\prime}\right)-(n-1)\left(\xi^{\prime}, \xi^{\prime}\right)=0
$$

Integrating $i(\xi)(D \Gamma \xi)$ on $M^{n}$, we have

$$
(\xi, D \Gamma \xi)=\left(\xi^{\prime}, D \xi\right)-\left(\xi^{\prime}, \xi^{\prime}\right)-(n-2)(\xi, \xi)
$$




$$
=-(n-2)(e(\eta) \xi, e(\eta) \xi) \text {. }
$$

Taking account of (2.13) again and considering Lemma 4.1, it follows that

$$
\begin{aligned}
(\Gamma \xi, \Gamma \xi) & =(\xi, D \Gamma \xi)-(n-1)(e(\eta) \xi, \Gamma \xi) \\
& =-(n-2)(e(\eta) \xi, e(\eta) \xi)+(n-1)(e(\eta) \xi, e(\eta) \xi) \\
& =(e(\eta) \xi, e(\eta) \xi)
\end{aligned}
$$

and the lemma is proved.

THEOREM 4.3. In a compact Sasakian space, $\bar{\xi}=\Phi \xi$ is a closed 1-form for any $C$-Killing form $\xi$.

PROOF. By virtue of Theorem 3.4, a 1 -form $\xi=\xi-\xi^{\prime} \eta$ is a special $C$-Killing form for a $C$-Killing form $\xi$. Moreover the relation

$$
\Phi \xi=\Phi \zeta
$$

holds good. Therefore it is sufficient to prove the theorem for a special $C$ Killing form $\xi$. From (4.3), (4.4) and (4.6), we have

$$
\begin{aligned}
(d \bar{\xi}, d \bar{\xi}) & =(\Gamma \xi, \Gamma \xi)+2(\Gamma \xi, e(\eta) \xi)+(e(\eta) \xi, e(\eta) \xi) \\
& =(e(\eta) \xi, e(\eta) \xi)-2(e(\eta) \xi, e(\eta) \xi)+(e(\eta) \xi, e(\eta) \xi) \\
& =0,
\end{aligned}
$$

which shows $d \bar{\xi}=0$.

From this Theorem 4.3 and (4.3), we have for a special $C$-Killing form $\xi$

$$
\begin{aligned}
& \nabla_{\lambda} \bar{\xi}_{\mu}=\nabla_{\mu} \bar{\xi}_{\lambda}, \\
& \Gamma \xi=-e(\eta) \xi
\end{aligned}
$$

Making use of (4.8), (4.2) becomes a simpler form as follows.

COROLlARY 4.4. In a compact Sasakian space, we have for a special C-Killing form $\xi$

$$
\nabla_{\lambda} \nabla_{\mu} \xi_{v}=R_{\mu \nu \lambda}^{\rho} \xi_{\rho}-2 \nabla_{\lambda} \bar{\xi}_{\mu} \eta_{\nu}+2 \xi^{\rho}\left(\varphi_{\rho \lambda} \varphi_{\mu \nu}+\varphi_{\rho \mu} \varphi_{\lambda v}\right)
$$

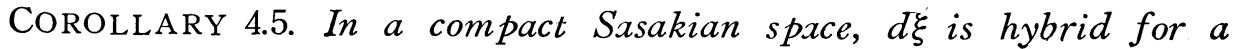

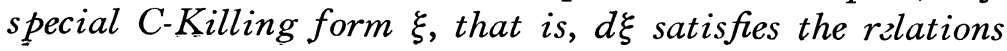




$$
\eta^{\rho}(d \xi)_{\rho \lambda}=0, \quad \varphi_{\lambda}{ }^{\rho} \varphi_{\mu}^{\sigma}(d \xi)_{\rho \sigma}=(d \xi)_{\lambda \mu} .
$$

PROOF. Since $i(\eta) d \xi=\theta(\eta) \xi-d i(\eta) \xi=0$, the first relation is evident. Next, (4.9) is written explicitely as follows

$$
\boldsymbol{\phi}_{\lambda}{ }^{\sigma} \nabla_{\sigma} \xi_{\mu}-\phi_{\mu}{ }^{\sigma} \nabla_{\sigma} \xi_{\lambda}=-\eta_{\lambda} \xi_{\mu}+\eta_{\mu} \xi_{\lambda}
$$

Transvecting it with $\varphi_{\nu}^{\lambda}$, we have

$$
\boldsymbol{\varphi}_{\lambda}{ }^{\rho} \boldsymbol{\varphi}_{\mu}{ }^{\sigma} \nabla_{\sigma} \xi_{\rho}=\left(\nabla_{\mu} \xi_{\lambda}-\nabla_{\lambda} \xi_{\mu}\right) / 2
$$

Exchanging the indices $\lambda$ and $\mu$ in this relation, and subtracting them, we get

$$
\boldsymbol{\varphi}_{\lambda}{ }^{\rho} \boldsymbol{\varphi}_{\mu}^{\sigma}\left(\nabla_{\sigma} \xi_{\rho}-\nabla_{\rho} \xi_{\sigma}\right)=\nabla_{\mu} \xi_{\lambda}-\nabla_{\lambda} \xi_{\mu}
$$

This is equivalent to

$$
\boldsymbol{\varphi}_{\lambda}{ }^{\rho} \boldsymbol{\varphi}_{\mu}{ }^{\sigma}(d \xi)_{\rho \sigma}=(d \xi)_{\lambda \mu}
$$

LEMMA 4.6. In a Sasakian space, we have for a 1-form $\xi$

$$
\begin{aligned}
& i(\xi) L-L i(\xi)=-2 e(\bar{\xi}), \quad \bar{\xi}=\Phi \xi, \\
& i(\xi) \Lambda=\Lambda i(\xi), \quad i(\eta) \Lambda_{\xi}=\Lambda_{\xi} i(\eta) .
\end{aligned}
$$

PROOF. For any $p$-form $u=\left(u_{\lambda_{1} \ldots \lambda_{p}}\right)$, we have

$$
\begin{aligned}
& \frac{1}{2}(i(\xi) L u)_{\lambda_{0} \ldots \lambda_{p}}=\xi^{\sigma}\left(\phi_{\sigma \lambda_{0}} u_{\lambda_{1} \ldots \lambda_{p}}-\sum_{i} \boldsymbol{\varphi}_{\sigma \lambda_{i}} u_{\lambda_{1} \ldots \hat{\lambda}_{0} \ldots \lambda_{p}}-\sum_{i} \phi_{\lambda_{i} \lambda_{0}} u_{\lambda_{1} \ldots \hat{\sigma} \cdots \lambda_{p}}^{i}\right. \\
& \left.+\sum_{i<j} \phi_{\lambda_{1} \lambda_{j}} u_{\lambda_{1} \ldots \hat{\sigma} \ldots \hat{\lambda}_{0} \cdots \lambda_{p}} \stackrel{i}{j}\right)=-\left(\bar{\xi}_{\lambda_{0}} u_{\lambda_{1} \ldots \lambda_{p}}-\sum_{i} \bar{\xi}_{\lambda_{i}} u_{\lambda_{1} \ldots \hat{\lambda}_{0} \cdots \lambda_{p}}\right) \\
& +\left(\phi_{\lambda_{0} \lambda_{i}} \xi^{\sigma} u_{\sigma \lambda_{2} \ldots \lambda_{p}}-\sum_{j \geqq 2} \phi_{\lambda_{0} \lambda_{j}} \xi^{\sigma} u_{\sigma \lambda_{2} \ldots \hat{\lambda}_{1} \cdots \lambda_{p}} \stackrel{j}{j}-\sum_{j \geqq 2} \phi_{\lambda_{j} \lambda_{1}} \xi^{\sigma} u_{\sigma \lambda_{2} \cdots \hat{\lambda}_{0} \cdots \lambda_{p}}\right. \\
& \left.+\sum_{2 \leqq i<j} \boldsymbol{\varphi}_{\lambda_{\mathfrak{s}} \lambda_{j}} \xi^{\sigma} u_{o \lambda_{2} \ldots \hat{\lambda}_{0} \ldots \hat{\lambda}_{1}^{\prime} \ldots \lambda_{p}}^{i}\right)=-\left(e(\overline{\xi)} u)_{\lambda_{0} \ldots \lambda_{p}}+\frac{1}{2}(\operatorname{Li}(\xi) u)_{\lambda_{0} \ldots \lambda_{p}} .\right.
\end{aligned}
$$

(4.12) is evident.

LEMMA 4.7. In a Sasakian space, we have for a 1-form $\xi$ 


$$
\triangle i(\xi)-i(\xi) \triangle=d \Lambda_{\xi}-\Lambda_{\xi} d+\delta \theta(\xi)-\theta(\xi) \delta
$$

This is easily obtained from (1.8).

LEMMA 4.8. In a compact Sasakian space, we have for a special $C$ Killing form $\xi$ and for any $p$-form $u$,

$$
\begin{gathered}
(\delta \theta(\xi)-\theta(\xi) \delta) u=2 e(\bar{\xi}) \wedge u+2 i(\bar{\xi}) i(\eta) d u-2 d i \overline{(\xi)} u^{\prime} \\
+4 \overline{\xi^{\rho}} \nabla_{\rho} u^{\prime}+2\left(D \xi-(n-1) \xi^{\prime}\right) u^{\prime}
\end{gathered}
$$

where we put $\bar{\xi}=\Phi \xi, \xi^{\prime}=i(\eta) \xi, u^{\prime}=i(\eta) u$,

PROOF. (4.14) is a result of a little complicated but straightforward calculation. We sketch the outline. For a $p$-form $u=\left(u_{\lambda_{1} \ldots \lambda_{p}}\right)$, we have

$$
\begin{gathered}
(\delta \theta(\xi) u)_{\lambda_{2} \cdots \lambda_{p}}=-\nabla^{\rho}\left(\xi^{\sigma} \nabla_{\sigma} u_{\rho \lambda_{2} \cdots \lambda_{p}}+\nabla_{\rho} \xi^{\sigma} u_{\sigma \lambda_{2} \cdots \lambda_{p}}+\sum_{i \geq 2} \nabla_{\lambda_{t}} \xi^{\sigma} u_{\sigma \lambda_{2} \cdots \hat{\sigma} \cdots \lambda_{p}}\right) \\
=A+B+C+D,
\end{gathered}
$$

where we put

$$
\begin{aligned}
& A=-\left(\nabla^{\rho} \xi^{\sigma}+\nabla^{\sigma} \xi^{\rho}\right) \nabla_{\rho} u_{\sigma \lambda_{2} \ldots \lambda_{p}}, \\
& B=-\nabla^{\rho} \nabla_{\rho} \xi^{\sigma} u_{\sigma \lambda_{2} \ldots \lambda_{p}}, \quad C=-\sum_{j \geqq 2} \nabla^{\rho} \nabla_{\lambda_{j}} \xi^{\sigma} u_{\rho \lambda_{2} \cdots \hat{\sigma} \cdots \lambda_{p}}, \\
& D=-\xi^{\sigma} \nabla^{\rho} \nabla_{\sigma} u_{\rho \lambda_{2} \ldots \lambda_{p}}-\sum_{j \geqq 2} \nabla_{\lambda_{j}} \xi^{\sigma} \nabla^{\rho} \nabla_{\rho} u_{\lambda_{2} \ldots \hat{\sigma} \cdots \lambda_{p}}^{j}
\end{aligned}
$$

Taking account of (3.7), we have

$$
\begin{aligned}
A & =-2 \xi^{\tau}\left({\varphi_{\tau}}_{\tau} \eta^{\sigma}+\boldsymbol{\varphi}_{\tau}^{\sigma} \eta^{\rho}\right) \nabla_{\rho} u_{\sigma \lambda_{2} \cdots \lambda_{p}} \\
& =2\left(i(\bar{\xi}) \nabla_{\eta} u\right)_{\lambda_{2} \ldots \lambda_{p}}+2 \overline{\xi^{\sigma}} \eta^{\rho} \nabla_{\sigma} u_{\rho \lambda_{2} \ldots \lambda_{p}} \\
& =2\left(i(\bar{\xi}) \nabla_{\eta} u\right)_{\lambda_{2} \ldots \lambda_{p}}+2 \overline{\xi^{\rho}} \nabla_{\rho} u_{\lambda_{2} \cdots \lambda_{p}}^{\prime}+2 \xi^{\prime} u_{\lambda_{2} \ldots \lambda_{p}}^{\prime}-2 \xi^{\sigma} u_{\sigma \lambda_{2} \ldots \lambda_{p}},
\end{aligned}
$$

and from (4.1) we obtain

$$
B=R_{\rho}^{\sigma} \xi^{\rho} u_{\sigma \lambda_{2} \cdots \lambda_{p}}+2\left(D \xi-(n+1) \xi^{\prime}\right) u_{\lambda_{2} \ldots \lambda_{p}}+4 \xi^{\sigma} u_{\sigma \lambda_{2} \cdots \lambda_{B^{\prime}}}
$$

By virtue of Corollary 4.4 and (4.8), $C$ can be calculated as 


$$
\begin{aligned}
& C=-\sum_{j \geqq 2}\left\{R_{\lambda_{j \sigma \rho}}{ }^{\varepsilon} \xi_{\varepsilon}-2 \nabla_{\rho} \overline{\xi_{\lambda}} \eta_{\sigma}+2 \xi^{\tau}\left(\boldsymbol{\varphi}_{\tau \rho} \boldsymbol{\varphi}_{\lambda_{\rho \sigma}}+\boldsymbol{\varphi}_{\tau \lambda_{j}} \boldsymbol{\varphi}_{\rho \sigma}\right)\right\} u_{\lambda_{2} \ldots}^{\stackrel{\rho}{\hat{\sigma}} \ldots \lambda_{\rho}}
\end{aligned}
$$

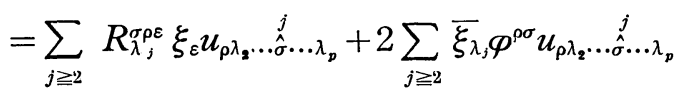

$$
\begin{aligned}
& +2 \sum_{j \geq 2}\left(\nabla_{\lambda_{j}} \bar{\xi}^{\rho} \eta^{\sigma}+\overline{\xi^{\rho}} \nabla_{\lambda_{j}} \eta^{\sigma}\right) u_{\rho \lambda_{2}} \ldots \hat{\sigma}_{\hat{\sigma} \ldots \lambda_{p}}^{j} \\
& =\sum_{j \geqq 2} R_{\lambda_{f}{ }^{\rho \varepsilon} \xi_{\varepsilon}} u_{\rho \lambda_{2} \cdots \hat{\sigma} \cdots \lambda_{p}}+2(e \overline{(\xi)} \Lambda u)_{\lambda_{2} \cdots \lambda_{p}}^{j}+2\left(\operatorname{di}(\bar{\xi}) u^{\prime}\right)_{\lambda_{2} \cdots \lambda_{p}} \\
& +2(i \overline{(\xi)}) i(\eta) d u)_{\lambda_{\mathbf{2}} \ldots \lambda_{p}}-2\left(i \overline{(\bar{\xi})} \nabla_{\eta} u\right)_{\lambda_{\mathbf{g}} \ldots \lambda_{p}}+2 \eta^{\sigma} \overline{\xi^{\rho}} \nabla_{\rho} u_{\sigma \lambda_{2} \ldots \lambda_{\nu}} .
\end{aligned}
$$

Lastly applying the Ricci's identity, we get

$$
\begin{aligned}
& D=-\xi^{\sigma}\left(\nabla_{\sigma} \nabla^{\rho} u_{\rho \lambda_{2} \ldots \lambda_{p}}+R_{\sigma}^{\varepsilon} u_{\varepsilon \lambda_{2} \ldots \Lambda_{p}}-\sum_{j \geqq \Perp} R_{\sigma \lambda_{j}}^{\rho} u_{\rho \lambda_{2} \ldots \hat{\varepsilon} \ldots \lambda_{p}}\right)+\sum_{j \geqq 2}^{j} \nabla_{\lambda_{j}} \xi^{\sigma}(\delta u)_{\lambda_{2} \ldots \hat{\sigma} \cdots \lambda_{p}} \stackrel{j}{j} \\
& =(e(\xi) \delta u)_{\lambda_{2} \ldots \lambda_{p}}-\xi^{\sigma} R_{\sigma}^{\varepsilon} u_{\varepsilon \lambda_{2} \ldots \lambda_{p}}-\sum_{j \geqq 2} \xi_{\varepsilon} R_{\lambda_{j}}^{\varepsilon \rho} u_{\rho} \lambda_{2} \ldots \hat{\sigma} \ldots \lambda_{p} .
\end{aligned}
$$

Adding these four relations, we can obtain (4.14).

Let $u$ be a $C$-harmonic $p$-form $(p \leqq(n-1) / 2)$, and $\xi$ be a special $C$-Killing form. Suppose that our Sasakian space is compact. Then by virtue of Proposition 1.1 and (4.14) we have

$$
(\delta \theta(\xi)-\theta(\xi) \delta) u=2 e(\bar{\xi}) \Lambda u .
$$

Taking account of Proposition 1.3 and Lemma 4.6, we get

$$
\begin{aligned}
i(\xi) \triangle u & =i(\xi) L \Lambda u=L i(\xi) \Lambda u-2 e \overline{(\xi}) \Lambda u \\
& =L \Lambda i(\xi) u-2 e(\bar{\xi}) \Lambda u .
\end{aligned}
$$

Since

$$
\begin{aligned}
\delta i(\xi) u & =\Lambda_{\xi} u-i(\xi) e(\eta) \Lambda u \\
& =\Lambda_{\xi} u-\xi^{\prime} \Lambda u+e(\eta) i(\xi) \Lambda u
\end{aligned}
$$

is valid and $\xi^{\prime}$ is constant, we have 
$4 \dot{8} 2$

Ý. OGAWA

$$
\begin{aligned}
\left(i(\xi) u, d \Lambda_{\xi} u\right) & =\left(\delta i(\xi) u, \Lambda_{\xi} u\right) \\
& =\left(\Lambda_{\xi} u, \Lambda_{\xi} u\right)-\xi^{\prime}\left(\Lambda u, \Lambda_{\xi} u\right) .
\end{aligned}
$$

Hence it follows from (4.13) that

$$
\begin{aligned}
(i(\xi) u, \triangle i(\xi) u) & \left.=(i(\xi) u, i(\xi) \triangle u)+\left(i(\xi) u, d \Lambda_{\xi} u\right)+(i(\xi) u, 2 e \bar{\xi}) \Lambda u\right) \\
& =(\Lambda i(\xi) u, \Lambda i(\xi) u)+\left(\Lambda_{\xi} u, \Lambda_{\xi} u\right)-\xi^{\prime}\left(\Lambda u, \Lambda_{\xi} u\right) .
\end{aligned}
$$

On the other hand, $\Lambda u$ is also $C$-harmonic from Proposition 1.2 and therefore

$$
d \Lambda u=0, \quad i(\eta) \Lambda u=0
$$

hold good. Hence we have

$$
\begin{aligned}
\left(\Lambda u, \Lambda_{\xi} u\right)= & (\Lambda u, \delta i(\xi) u+i(\xi) e(\eta) \Lambda u) \\
& =\left(\Lambda u, \xi^{\prime} \Lambda u-e(\eta) i(\xi) \Lambda u\right)=\xi^{\prime}(\Lambda u, \Lambda u)
\end{aligned}
$$

Using this relation and (4.15), it follows that

$$
\begin{aligned}
(\delta i(\xi) u, \delta i(\xi) u) & =\left(\Lambda_{\xi} u, \Lambda_{\xi} u\right)+\xi^{\prime 2}(\Lambda u, \Lambda u)+(\Lambda i(\xi) u, \Lambda i(\xi) u)-2 \xi^{\prime}\left(\Lambda u, \Lambda_{\xi} u\right) \\
& =\left(\Lambda_{\xi} u, \Lambda_{\xi} u\right)+(\Lambda i(\xi) u, \Lambda i(\xi) u)-\xi^{\prime}\left(\Lambda u, \Lambda_{\xi} u\right) .
\end{aligned}
$$

Therefore we can obtain

$$
(i(\xi) u, \triangle i(\xi) u)=(\delta i(\xi) u, \delta i(\xi) u),
$$

which shows that $d i(\xi) u=0$. Hence we have $\theta(\xi) u=0$.

For an arbitrary scalar function $f$, the Lie derivative of any $C$-harmonic $p$-form $u(p \leqq(n-1) / 2)$ with respect to $f \eta$ vanishes. In fact, we have

$$
\theta(f \eta) u=f \theta(\eta) u+e(d f) i(\eta) u=0,
$$

if $p \leqq(n-1) / 2$. Taking account of Theorem 3.4, we decompose a $C$-Killing form $\xi$ as the sum of a special $C$-Killing form $\zeta$ and $\xi^{\prime} \eta$. Then we have

$$
\theta(\xi) u=\theta(\zeta) u+\theta\left(\xi^{\prime} \eta\right) u=0
$$

for any $C$-harmonic $p$-form $u$. Consequently we attained to the following

THEOREM 4.9. In a compact $n$-dimensional Sasakian space, let $u$ be a 
$C$-harmonic pform $(p \leqq(n-1) / 2)$ and $\xi$ be a C-Killing form, then we have

$$
\theta(\xi) u=0 .
$$

Especially, a harmonic $p$-form $(p \leqq(n-1) / 2)$ is $C$-harmonic in compact case. Further the fundamental 2 -form $d \eta$ is $C$-harmonic. Thus we have the following corollaries.

COROLlARY 4.10. Let $u$ be a harmonic $p$-form $(p \leqq(n-1) / 2)$ and $\xi$ be a C-Killing form. Then we have

$$
\theta(\xi) u=0
$$

COROllaRy 4.11. Let $\xi$ be a C-Killing form. Then it satisfies

$$
\theta(\xi) d \eta=0
$$

5. Regular Sasakian structure. We consider the meaning of $C$-Killing forms on a compact Sasakian space which has regular structure. Let $\left(M^{n}, p\right.$, $B^{n-1}$ ) be a fibration of Boothby-Wang, where $B^{n-1}=M^{n} /(\eta)$ is the base space and $p: M^{n} \rightarrow B^{n-1}$ is the projection. We denote by $\nabla, \nabla^{\prime}$ the covariant differentiations with respect to the Riemann metric $g_{\lambda \mu}$ on $M^{n}$ and $g_{a b}^{\prime}{ }^{1)}$ on $B^{n-1}$ defined naturally by $g_{\lambda_{\mu}}$. We fix a point $x \in M^{n}$ and set $y=p(x)$. Taking local coordinates systems $\left(x^{\lambda}\right)$ and $\left(y^{a}\right)$ arround $x$ and $y$, we represent the projection mapping as

$$
y^{a}=p^{a}\left(x^{1}, \cdots, x^{n}\right) .
$$

We put $p_{\lambda}^{\alpha}=\partial p^{\alpha} / \partial x^{\lambda}$, and $\bar{u}=p^{*} u=\left(\bar{u}_{\lambda_{1} \ldots \lambda_{p}}\right)$ for a $p$-form $u=\left(u_{a_{1} \cdots a_{p}}\right)$ on $B^{n-1}$. Then we know that the following relation

$$
\nabla_{\mu} \bar{u}_{\lambda_{1} \cdots \lambda_{p}}=p_{\lambda_{1}}^{a_{1}} \cdots p_{\lambda_{p}^{p}}^{a_{p}} p^{b}{ }_{\mu} \nabla^{\prime}{ }_{b} u_{a_{1} \cdots a_{p}}-\sum_{i}\left(\boldsymbol{\phi}_{\mu}{ }^{\rho} \eta_{\lambda_{t}}+\boldsymbol{\phi}_{\lambda_{i}}{ }^{\rho} \eta_{\mu}\right) \bar{u}_{\lambda_{1} \cdots \hat{\rho} \ldots \lambda_{p}} \stackrel{i}{i}
$$

is valid [4]. Applying (5.1) to a 1 -form $u_{a}$, we have

$$
\nabla_{\mu} \bar{u}_{\lambda}=p^{a}{ }_{\lambda} p_{\mu}{ }_{\mu} \nabla^{\prime}{ }_{b} u_{a}-\left(\phi_{\mu}{ }^{\rho} \eta_{\lambda}+\varphi_{\lambda}{ }^{\rho} \eta_{\mu}\right) \bar{u}_{\rho} .
$$

Now suppose that the 1 -form $u_{a}$ is a Killing form on $B^{n-1}$. Then (5.2) shows

1) The Latin indices $a, b, \ldots$, run from 1 to $n-1$. 


$$
\begin{aligned}
\nabla_{\mu} \bar{u}_{\lambda}+\nabla_{\lambda} \bar{u}_{\mu} & =p^{a}{ }_{\lambda} p^{b}{ }_{\mu}\left(\nabla^{\prime}{ }_{a} u_{b}+\nabla^{\prime}{ }_{b} u_{a}\right)-2\left(\varphi_{\mu}{ }^{\rho} \eta_{\lambda}+\varphi_{\lambda}{ }^{\rho} \eta_{\mu}\right) \vec{u}_{\rho} \\
& =2 \bar{u}^{\circ}\left(\phi_{\rho \lambda} \eta_{\mu}+\varphi_{\rho \mu} \eta_{\lambda}\right) .
\end{aligned}
$$

Thus the form $\bar{u}=p^{*} u$ satisfies (3.7) and as the space is compact, $u$ is a special $C$-Killing form.

Conversely we consider a $C$-Killing form $\xi$ which satisfies $i(\eta) \xi=0$. Since $\theta(\eta) \xi_{\lambda}=0$ from Lemma 3.1, there exists a 1 -form $u$ on $B^{n-1}$ such that $\xi=p^{*} u$. Then we have by virtue of (3.7) and (5.2)

$$
\begin{aligned}
p_{\mu}^{b} p_{\lambda}^{a}{ }_{\lambda}\left(\nabla_{a}^{\prime} u_{b}+\nabla_{b}^{\prime} u_{a}\right) & =\nabla_{\mu} \xi_{\lambda}+\nabla_{\lambda} \xi_{\mu}-2 \xi^{\rho}\left(\varphi_{\rho \lambda} \eta_{\mu}+\varphi_{\rho \mu} \eta_{\lambda}\right) \\
& =0
\end{aligned}
$$

Therefore we see

$$
\nabla_{b}^{\prime} u_{a}+\nabla_{a}^{\prime} u_{b}=0
$$

which shows that $u_{a}$ is a Killing form on $B^{n-1}$. Thus we have the following

THEOREM 5.1. Let $M^{n}$ be a compact regular Sasakian space, and $B^{n-1}$ be the base space of the fibration of Boothby-Wang. Then the vector space of Killing 1-forms on $B^{n-1}$ is isomorphic to the vectcr space of C-Killing forms on $M^{n}$ which are orthogonal to $\eta$.

\section{BIBLIOGRAPHY}

[1] W. M. Bоотнву AND H.C. WANG, On contact manifolds, Ann. Math., 68(1958), 721734.

[2] S. TAChiBAnA, On harmonic tensors in compact Sasakian spaces, Tôhoku Math. Journ., $17(1965), 271-284$.

[3] S. TACHIBANA, On a decomposition of $C$-harmonic forms in a compact Sasakian space, Tôhoku Math. Journ., 19(1967), 198-212.

[4] Y. OGAWA, On C-harmonic forms in a compact Sasakian space, Tôhoku Math Journ. 19 (1967), 267-296.

[5] S.SASAKI, On differentiable manifolds with certain structures which are closely related to almost contact structure, I, Tôhoku Math. Journ., 12(1960), 459-476.

[6] T. FuJiTANI, Complex valued differential forms on normal contact Riemannian manifolds, Tôhoku Math. Journ., 18(1966), 349-361.

[7] S. I. Goldberg, Curvature and Homology, Academic Press, New York, 1962.

DEPARTMENT OF MATHEMATICS

OCHANOMIZU UNIVERSITY

TOKYO, JAPAN 